EPJ Web of Conferences 82, 01051 (2015)

DOI: $10.1051 /$ epjconf/20158201051

(C) Owned by the authors, published by EDP Sciences, 2015

\title{
Automated resource-saving technology of ion-exchange water treatment
}

\author{
M. Livshits \\ Doctor of Science, Samara State Technical University, Russia
}

\begin{abstract}
Stable high quality of the purified water can be provided by adaptive control of water-treatment installations with the observer in a loop of the control system on the basis of observer of ion exchange processes. To obtain this goal the following problems have been solved: the hierarchic structure of water treatment system is developed; the system of water treatment quality criteria for ion exchange processes is developed; the created mathematical model of ionic exchange processes is functionally oriented to application in control system as an observer; methodologies of identification of a mathematical model of ionic exchange processes is developed; verification of the mathematical model of ionic exchange is performed on experimental-industrial basis; automatic control system of water treatment with observer in the loop is developed for low-waste installation of a heat supply system.
\end{abstract}

Water resources that are widely used in the modern industry rarely meet the requirements of direct applications. Impurities contained in natural water are removed at water treatment installations (WTI) implementing technological processes chain, and ensuring water quality that is relevant to the technical regulations, sanitary rules and norms. This leads to the important role of the WTI as a subsystem in the system structure of the production and consumption of heat and electricity.

Decreasing in the quality of treated water leads to huge costs for the restoration of the main energy-producing equipment - steam generators, turbines, and consumers' technology systems - heat exchangers, pipelines. Costs for improvements of WTI are significantly lower than the cost of liquidation of technological failures consequences.

The application of currently developed technologies for the production of purified water, consisting of the processes for removal of suspended, colloidal, molecular-dispersed impurities and gases is constrained by the instability and uncertainty in source water quality, uncertainty of market demands and technological modes instability.

The problem solution can be based on system analysis using a systematic structural mathematical modeling and identification of water treatment processes with the help of observer in the circuit of the automatic control system (ACS) of WTI in order to provide an effective control under conditions of uncertainty [1-3]. Figure 1 presents a structural diagram of an adaptive ACS for regulating the quality of feed water at large thermal power plants.

The loop of the ACS contains the observer which is based on functionally oriented mathematical model (FOM) of ion-exchange water treatment and includes also a mathematical model of the $\mathrm{pH}$ index to determine the components of alkalinity; mathematical model of the ion couples concentration;

This is an Open Access article distributed under the terms of the Creative Commons Attribution License 4.0, which permits unrestricted use, distribution, and reproduction in any medium, provided the original work is properly cited. 


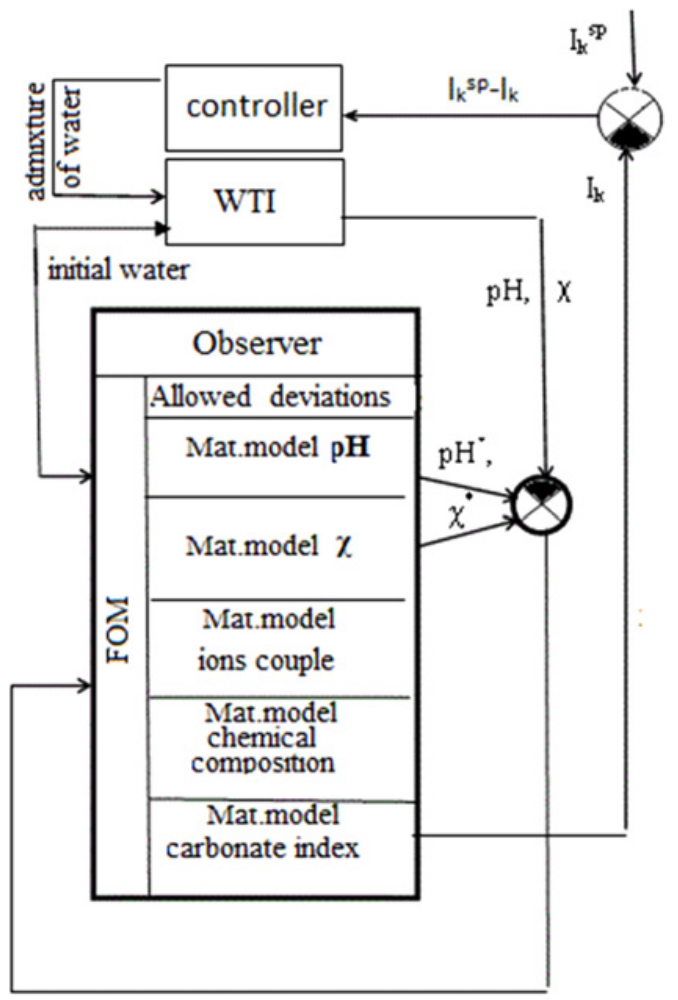

Figure 1. Block diagram of the adaptive ACS for control of water treatment quality.

the mathematical model of electrical conductivity $\chi_{\mathrm{n}}$; mathematical model of chemical analysis; mathematical model of carbonate index.

Main indicators of corrosion-scale properties of water are are structured and considered as quality criteria of chemical water treatment.

These criteria are traditionally determined by appropriate experimental analysis of mineralization components. As a result, the mode correction is delayed and it leads to a loss in reliability and WTI efficiency, and increasing the risk of failures and accidents. Application of observer in the loop of ACS allows solving that problem.

The problem of the development and identification of FOM is formulated and solved. The process of ion exchange is represented as a controlled object in a rather general form: $\left(\overline{\mathbf{C}_{\mathbf{r}}}\right)_{\mathbf{n}}=\mathbf{F}_{\mathbf{0}}^{\mathbf{T}}(\overline{\mathbf{u}})+\mathbf{v}_{\mathbf{0}}$, where $\mathbf{F}_{\mathbf{0}}(\overline{\mathbf{u}})$ is a link vector - operator, $\mathbf{v}_{\mathbf{0}}^{-}$is a random error, $\overline{\mathbf{u}}$-is the control vector, $\left(\overline{\mathbf{C}_{\mathbf{r}}}\right)_{\mathbf{n}}=$ $\left[\left(\mathbf{C}_{\mathbf{1}}\right)_{\mathbf{n}},\left(\mathbf{C}_{2}\right)_{\mathbf{n}}, \ldots,\left(\mathbf{C}_{\mathbf{r}}\right)_{\mathbf{n}}, \ldots\left(\mathbf{C}_{\mathbf{R}}\right)_{\mathbf{n}}\right]^{T}-$ is the chemical composition of the $\mathrm{n}$-th flow.

Vector $\left(\overline{\mathbf{C}_{\mathbf{r}}}\right)_{\mathbf{n}}$ is determined by observation vector $\overline{C_{H}}$, during the identification process, with noisyobservations error: $\mathbf{v}_{\mathbf{H}}$ :

$$
\left(\overline{\mathbf{C}}_{\mathbf{r}}^{*}\right)_{\mathbf{n}}=\mathbf{F}_{\mathbf{H}} \mathbf{T}^{\left.\overline{\left(\mathbf{C}_{\mathbf{H}}\right.}\right)_{\mathbf{n}}}+\mathbf{v}_{\mathbf{H}} .
$$

In the water treatment technology based on ion-exchange process the parameters $\boldsymbol{p} \boldsymbol{H}_{\boldsymbol{n}}$ and $\mathbf{v}_{n}$ can be usually considered as continuously observable indicators of a process quality (see Fig. 1). In this case Equation (1) takes the following form: $\left(\overline{\boldsymbol{C}_{\boldsymbol{r}}^{*}}\right)_{\boldsymbol{n}}=\mathbf{F}_{\mathbf{H}}\left(\boldsymbol{p} \boldsymbol{H}_{\boldsymbol{n}} \cdot \chi_{\boldsymbol{n}}\right)^{\boldsymbol{T}}+\left(\mathbf{I}_{\mathbf{p H}} \cdot \mathbf{I}_{\chi}\right)^{\mathbf{T}}$, where: $\boldsymbol{l}_{\boldsymbol{p} \boldsymbol{H}}$ - error of $\mathbf{p} \mathbf{H}_{\mathbf{n}}$ observation; $\mathbf{I}_{\chi}-$ error of $\boldsymbol{\chi}_{\mathbf{n}}$ observation; $\boldsymbol{F}_{\mathbf{H}}-\mathrm{n}$ asymptotical observer implemented within a given time $0<\tau_{\mathrm{sp}}<\infty$ for which the following condition: $\lim _{\tau \rightarrow \tau_{s p}} \|\left(\overline{\left.\mathbf{C}_{\mathbf{r}}^{*}\right)_{\mathbf{n}}}--\left(\overline{\mathbf{C}_{\mathbf{r}}}\right)_{\mathbf{n}} \| \leq \varepsilon_{s p}\right.$, is true. 
a

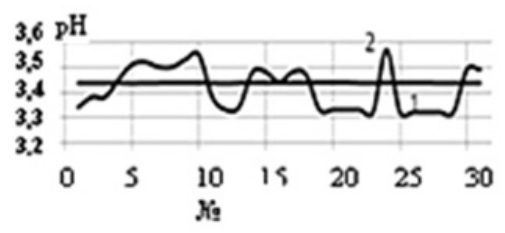

$\mathrm{b}$
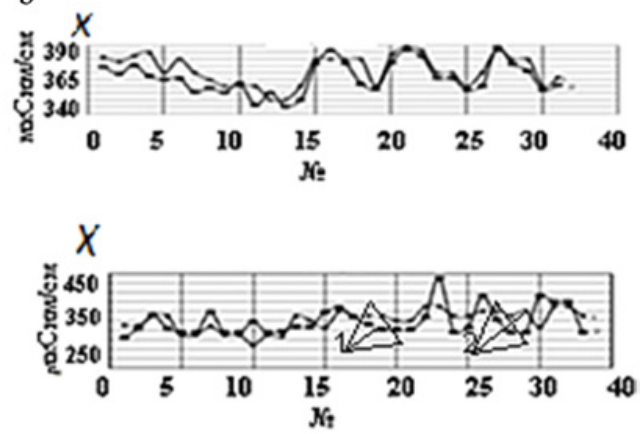

Figure 2. a) Model of $\mathrm{pH}, \mathrm{b}$ ) model $\chi, 1$ - experiment; 2 - calculations.
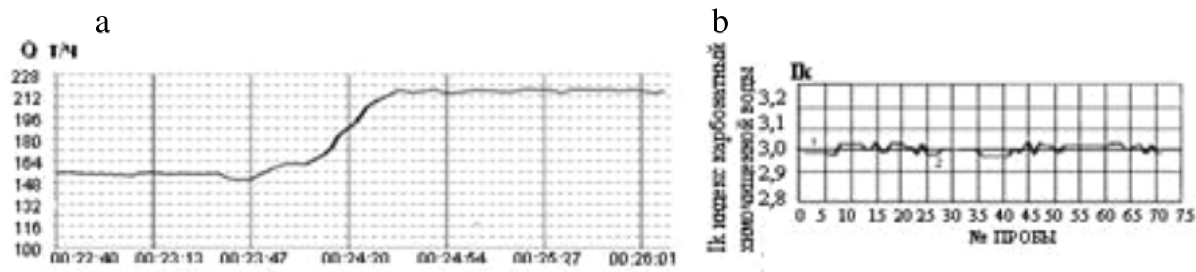

Figure 3. Stabilization of carbonate index in ACS a-raw water to mix, b-carbonate index.

Here: $\varepsilon_{s p}$ is prescribed admissible error. The problem of identification of the mineralization components concentrations which cannot be directly measured is formulated and solved. The integral quality indexes $p H$ and $\chi$ are used in the identification procedure The structure of the FOM (see Fig. 1) is based on the developed by the author methods for computations of $\mathrm{pH}$ and components alkalinity, ion pairs conductivity, mineralization components using a priori information about the flow. Figures 2, 3 demonstrate the good quality of the simulation and the ACS operation.

Spent solutions formed during the regeneration of H-cationite filters are always supersaturated in comparison with calcium sulphate. Installation of processing and recycling of wastewater provides reusable solutions that dramatically reduces the resource consumption of the WTI.

\section{References}

[1] M. Livshits, Differential Equations and Their Applications, Bulletin of the SSTU, Series Mathematical, 4, 45 (2007)

[2] M. Livshits, Yu. Solodyanikova. Proc. VIII International Conference, Recent advances in science and education, 36 (2013)

[3] M. Livshits, V. Solodyannikov, Yu. Solodyannikova, Energy saving and water treatment, 5, 9 (2009) 